\title{
sciendo
}

\author{
BULGARIAN ACADEMY OF SCIENCES
}

CYBERNETICS AND INFORMATION TECHNOLOGIES • Volume 20, No 2

Sofia $\bullet 2020 \quad$ Print ISSN: 1311-9702; Online ISSN: 1314-4081

DOI: $10.2478 /$ cait-2020-0015

\section{Fuzzy Group Full Consistency Method for Weight Determination}

\section{Galina Ilieva}

University of Plovdiv Paisii Hilendarski, Plovdiv, Bulgaria

Institute of Information and Communication Technologies, Bulgarian Academy of Sciences, 1113 Sofia, Bulgaria

E-mail: galili@uni-plovdiv.bg

Abstract: In this paper, the FUll COnsistency Method (FUCOM) is extended to work in a collective manner, to solve a fuzzy optimization problem and to obtain the fuzzy weights of criteria. The employment of a predefined order of criteria decreases the number of fuzzy comparisons needed in the evaluation phase. The defuzzified values of the optimal weight coefficients are calculated by Graded Mean Integration Representation formula. This feature also reduces time complexity without affecting the quality of the solution. Two practical examples are presented to verify the reliability and feasibility of the proposed fuzzy group FUCOM. The obtained results demonstrate that the new fuzzy group weight determination method can obtain appropriate criteria importance.

Keywords: Multi-criteria decision methods, determination of fuzzy weight coefficients, fuzzy optimization, fuzzy group full consistency method.

\section{Introduction and related works}

Assessment of criteria weights is an essential and integral part of the Multi-Criteria Decision-Making (MCDM) process. The methods for determining the weights of the criteria are based on their relative significance according to the preferences of experts. In case of uncertain and ambiguous data, the complexity of the problem increases and requires new instruments and tools.

Many scholars have proposed various MCDM algorithms [9]. Due to the subjectivism and the uncertainty in evaluation process, using fuzzy numbers and their varieties is a possible approach in decision-making problems via triangular [6], hesitant [11], and interval-valued [19] fuzzy numbers. Many fuzzy MCDM have been developed and employed, such as aggregations [17] of fuzzy relations [14] with fuzzy criteria weights [15] and fuzzy weighting functions [16], fuzzy EDAS [8], fuzzy TOPSIS [20].

The multiple applications of new MCDM modifications in green supplier selection [26] are being exhaustively researched, as well as those in bidding strategies selection [7], economic clusters ranking [18], network selection [21], technology 
ordering [4], and factor selection while assessing technological innovations in micro, small and medium enterprises [23].

Many researchers have designed MCDM algorithms for evaluating relative weight values. A comprehensive review of different studies on calculating relative importance of criteria can be found in [9]. FUll COonsistency Method (FUCOM) is an indirect and subjective method, in which experts assess criteria significance according to their own point of view. FUCOM is based on predefined priority and pairwise comparison of criteria and, therefore, only a small number of comparisons are needed. Another feature of this method is its built-in ability for model verification. Model consistency is guaranteed via the satisfaction of the transitivity condition [13].

Applications of FUCOM are abundant and prove its versatility in solving a wide gamut of tasks, such as selecting contractors for solar panel installations [2], ranking airlines [1], choosing forklifts [5], urban mobility system [12] and human resource evaluation [22]. In [2] a hybrid (SWARA and FUCOM) method was created, representing criteria weights as grey numbers. In [1] the criteria weights were determined using FUCOM method and results were compared to those obtained by AHP method. The best alternatives were selected via hybrid FUCOM-WASPAS model and authors' choice was conditioned by the optimality of criteria weights [5]. In [12] a MCDM based fuzzy FUCOM-Dombi-Bonferroni model is proposed for evaluating urban mobility systems. A FUCOM-MARCOS model was introduced for the purpose of human resource evaluation, on which the overall efficiency of the company depends [22].

The idea of developing and applying fuzzy sets for weight determination is not new in soft computing. Since decision makers often encounter vague and uncertain cases when calculating the relative importance of one element to another, linguistic values can be given instead of crisp assessments. In [24] the authors suggested an approach based on fuzzy sets to handle uncertainties of AHP in fuzzy environments. The new fuzzy model combines the fuzzy linguistic aspect of fuzzy theory with DEMATEL algorithm in [25]. In [3], the entropy method was extended for fuzzy sets based on triangular fuzzy membership functions. In [6], a modified best-worst method (BWM) was applied via triangular fuzzy numbers.

The purpose of this paper is to present a fuzzy group FUCOM [13], based on imprecise evaluations of the priorities among compared criteria. FUCOM is a relatively new multi-criteria method for determining weight coefficients, which is particularly attractive in the presence of a priori information about ratios between criteria importance.

In order to extend the application of FUCOM to weight coefficient evaluation in an environment of uncertainty, we create an algorithm for collective determination of criteria weights via triangular fuzzy numbers. Section 2 describes the basic concept of classic FUCOM algorithm. Section 3 presents the fuzzy group FUCOM, which applies a specific defuzzification rule. Section 4 verifies new method using examples for weight assessments. Finally, the last section concludes with a summary of fuzzy group FUCOM and lists some future directions. 


\section{Classic FUCOM}

Let us consider a MCDM problem having $m$ alternatives $A=\left(A_{1}, A_{2}, \ldots, A_{m}\right)$ and $n$ decision criteria $C=\left(C_{1}, C_{2}, \ldots, C_{n}\right)$. The decision matrix $X=\left(x_{i j}\right)_{m \times n}$ shows all appraisals of the alternatives for each criterion. Let the vector of relative weights of all criteria be denoted as $w=\left(w_{1}, w_{2}, \ldots, w_{n}\right)$. The task is determining the values of the vector of relative weights of criteria $w$.

\section{Algorithm 1 [13].}

Step 1. Build the ranking set from the given evaluation criteria $C=\left(C_{1}, C_{2}, \ldots, C_{n}\right)$. The criteria ordering according to their expected significance is obtained as follows:

$$
C_{j(1)}>C_{j(2)}>\ldots>C_{j(k)},
$$

where $k$ represents the order of the observed criterion.

Step 2. Compare each pair of adjacent criteria and determine the comparative priorities $\varphi_{(k-1) / k}, k=1,2, \ldots, n$. Here $\varphi_{(k-1) / k}$ represents the importance of criterion $\mathrm{C}_{j(\mathrm{k}-1)}$ compared to that of criterion $\mathrm{C}_{j(\mathrm{k})}$, where $k-1, k$ represent the order of criteria. Then, derive the vector of comparative priorities of the evaluation criteria as follows:

$$
\Phi=\left(\varphi_{1 / 2}, \varphi_{2 / 3}, \ldots, \varphi_{(k-1) / \mathrm{k}}\right) .
$$

Step 3. Calculate the final values of the weight coefficients $\left(w_{1}, w_{2}, \ldots, w_{n}\right)$. They should satisfy the following two conditions:

a) The ratio of weight coefficients is equal to the comparative priority $\varphi_{(k-1) / k}$, (as defined in Step 2). In other words,

$$
\frac{w_{k-1}}{w_{k}}=\varphi_{(k-1) / k)} \text {. }
$$

b) The weight coefficients should satisfy the transitivity condition, i.e., $\varphi_{(k-2) /(k-1)} \otimes \varphi_{(k-1) / k}=\varphi_{(k-2) / k}$. Therefore, the second condition that the coefficients need to meet is

$$
\frac{w_{k-2}}{w_{k}}=\varphi_{(k-2) /(k-1)} \otimes \varphi_{(k-1) / \mathrm{k}}
$$

Based on the listed considerations, a nonlinear constrained programming model is built as follows:

$$
\begin{gathered}
\min \chi, \\
\text { s.t. }\left|\frac{w_{(k-1)}}{w_{k}}-\varphi_{(k-1) / k}\right| \leq \chi,\left|\frac{w_{k-2}}{w_{k}}-\varphi_{(k-2) /(k-1)} \otimes \varphi_{(k-1) / k}\right| \leq \chi, \\
\sum_{j=1}^{n} w_{j} \forall j, w_{j} \geq 0 \forall j .
\end{gathered}
$$

Solving that model produces the optimal values of the evaluation weights $\left(w_{1}, w_{2}, \ldots, w_{n}\right)$.

\section{The fuzzy group FUCOM}

Let there be $n$ criteria for evaluating a research object. Let $\tilde{a}_{i j}$ represent the relative fuzzy preference of criterion $i$ to criterion $j$, which is a triangular fuzzy number and $\tilde{a}_{i j}=(1,1,1)$ when $i=j$. According to FUCOM [13] and definitions of fuzzy operations [10], a pairwise comparison of two fuzzy weights is defined as a fuzzy 
comparison $\tilde{a}_{i j}=\widetilde{w}_{i} / \widetilde{w}_{j}$.

In Algorithm 2 there is a detailed description of fuzzy group FUCOM for calculating the fuzzy weight coefficients of criteria.

\section{Algorithm 2}

Step 1. The evaluation criteria $C=\left(C_{1}, C_{2}, \ldots, C_{n}\right)$ are ranked. The ranking is performed according to the significance of the criteria:

$$
C_{j(1)} \geq C_{j(2)} \geq \ldots \geq C_{j(\mathrm{k})},
$$

where the second index represents the rank of the criterion.

Step 2. A comparison of the ranked criteria is carried out and the fuzzy comparative priority $\tilde{\varphi}_{(k-1) / k}, k=1,2, \ldots, n$, where $k$ represents the rank of the criterion, is determined by each member of a group of experts. The mean values of fuzzy priorities generated by the team are calculated. The vector of the average fuzzy comparative priorities of the evaluation criteria is obtained as follows:

$$
\widetilde{\Phi}=\left(\tilde{\varphi}_{1 / 2}, \tilde{\varphi}_{2 / 3}, \ldots, \tilde{\varphi}_{(k-1) / k}\right) .
$$

Step 3. The optimal values of the fuzzy weight coefficients $\widetilde{w}=\left(\widetilde{w}_{1}, \widetilde{w}_{2}, \ldots, \widetilde{w}_{j}, \ldots, \widetilde{w}_{n}\right)$ are calculated, where $\widetilde{w}_{j}=\left(l_{j}, m_{j}, u_{j}\right), j=1,2, \ldots, n$. In order for the fully consistency condition to be met, the relative importance coefficients should satisfy the following two conditions.

a) The ratios of the fuzzy weight coefficients are equal to the corresponding fuzzy comparative priorities among $\tilde{\varphi}_{(k-1) / k}, k=1,2, \ldots, n$, calculated in Step 2:

$$
\frac{\widetilde{w}_{k-1}}{\widetilde{w}_{k}}=\tilde{\varphi}_{(k-1) / k} \text {. }
$$

b) The values of the fuzzy weight coefficients should satisfy the transitivity condition, i.e., $\tilde{\varphi}_{(k-2) /(k-1)} \otimes \tilde{\varphi}_{(k-1) / k}=\tilde{\varphi}_{(k-2) / k}$ or in our case:

$$
\frac{\widetilde{w}_{k-2}}{\widetilde{w}_{k}}=\tilde{\varphi}_{(k-2) /(k-1)} \otimes \tilde{\varphi}_{(k-1) / k} .
$$

Then a fuzzy nonlinear programming model can be defined as follows:

$$
\left|\frac{\widetilde{w}_{k}-1}{\widetilde{w}_{k}}-\tilde{\varphi}_{(k-1) / k}\right| \leq \tilde{\chi} \text {, and }\left|\frac{\widetilde{w}_{k-2}}{\widetilde{w}_{k}}-\widetilde{\varphi}_{(k-2) /(k-1)} \otimes \tilde{\varphi}_{(k-1) / k}\right| \leq \tilde{\chi},
$$

with the minimization of the value $\tilde{\chi}$, where $\tilde{\chi}=\left(l^{\chi}, m^{\chi}, u^{\chi}\right)$.

The final model for determining the optimal values of the fuzzy weight coefficients can be described as follows:

$$
\begin{gathered}
\text { s.t. }\left|\frac{\widetilde{w}_{k-1}}{\widetilde{w}_{k}}-\tilde{\varphi}_{(k-1) / k}\right| \leq \tilde{\chi},\left|\frac{\widetilde{w}_{k-2}}{\widetilde{w}_{k}}-\tilde{\varphi}_{(k-2) /(k-1)} \otimes \tilde{\varphi}_{(k-1) / k}\right| \leq \tilde{\chi}, \\
\sum_{j=1}^{n} R\left(\widetilde{w}_{j}\right)=1, l_{j} \leq m_{j} \leq u_{j}, l_{j} \geq 0 \forall j .
\end{gathered}
$$

Considering $l^{\chi} \leq m^{\chi} \leq u^{\chi}$, we let $\tilde{\chi}^{*}=\left(p^{*}, p^{*}, p^{*}\right) \leq l^{\chi}$, Equation (10) can be rearranged as follows:

$$
\begin{gathered}
\min \tilde{\chi}^{*}, \\
\text { s.t. }\left|\frac{\widetilde{w}_{k-1}}{\widetilde{w}_{k}}-\tilde{\varphi}_{(k-1) / k)}\right| \leq\left(p^{*}, p^{*}, p^{*}\right), \\
\left|\frac{\widetilde{w}_{k-2}}{\widetilde{w}_{k}}-\widetilde{\varphi}_{(k-2) /(k-1)} \otimes \tilde{\varphi}_{(k-1) / k}\right| \leq\left(p^{*}, p^{*}, p^{*}\right), \\
\sum_{j=1}^{n} G\left(\widetilde{w}_{j}\right)=1, \quad l_{j} \leq m_{j} \leq u_{j}, \quad l_{j} \geq 0 \quad \forall j .
\end{gathered}
$$

By solving Equation (11), the optimal fuzzy weights $\left(\widetilde{w}_{1}^{*}, \widetilde{w}_{2}^{*}, \ldots, \widetilde{w}_{n}^{*}\right)$ are obtained. 
The new elements in fuzzy group FUCOM are as follows:

1. Applying a set of linguistic terms or their equivalent fuzzy triangular numbers for measuring the significance of criteria, instead of crisp values (Step 2). (Step 2).

2 . Defining the average fuzzy criteria priority in case of group decision-making

3. Simplifying the heuristic formula is for converting fuzzy numbers to real values (Step 3 and Graded Mean Integration Representation (GMIR) formula [26]).

4. Reducing the number of constraints in comparison with other fuzzy triangular methods for weights determination (Step 3 and Equation (11)).

The advantages of the proposed fuzzy group FUCOM are the following:

- It works with group assessments in terms of both beneficial and cost criteria in a fuzzy environment;

- It decreases time complexity by using an heuristic formula for transforming fuzzy assessments to crisp ones;

- It reduces the number of nonlinear constraints by simplifying the optimization model.

\section{Practical examples}

Let fuzzy pairwise comparisons of criteria be performed by a group of decision makers via five grade linguistic variables and their corresponding Triangular Fuzzy Numbers (TFNs) [6].

Example 1. A buyer uses five criteria $C_{i}, i=1,2, \ldots, 5$, where $C_{1}$ is quality, $C_{2}$ - price, $C_{3}$ - comfort, $C_{4}$ - safety, and $C_{5}$ - style, to evaluate car alternatives, and then make a consumer buying decision. The fuzzy group FUCOM is used to select the optimal weight coefficients, which can reflect uncertainty and ambiguity of buyer when making the purchase decision. The vectors of fuzzy comparative priorities are given in Table 1 [6].

Table 1. Fuzzy comparative priorities (Example 1)

\begin{tabular}{|c|c|c|c|c|c|}
\hline Criteria & $C_{2}$ & $C_{1}$ & $C_{3}$ & $C_{4}$ & $C_{5}$ \\
\hline$\tilde{\varphi}_{(k-1) / k}$ & $(1,1,1)$ & $(0.67,1,1.5)$ & $(0.67,1,1.5)$ & $(1.5,2,2.5)$ & $(1.5,2,2.5)$ \\
\hline$\tilde{\varphi}_{(k-2) / k}$ & & & $(0.67,1,1.5)$ & $(1.5,2,2.5)$ & $(3.5,4,4.5)$ \\
\hline
\end{tabular}

Taking into consideration the fuzzy group FUCOM for getting the optimal fuzzy weights of all criteria, the following nonlinear constrained optimization problem can be built:

$$
\begin{gathered}
\min \tilde{\chi}^{*}, \\
\left|\frac{\widetilde{w}_{2}}{\widetilde{w}_{1}}-(0.67,1,1.5)\right| \leq\left(p^{*}, p^{*}, p^{*}\right),\left|\frac{\widetilde{w}_{1}}{\widetilde{w}_{3}}-(0.67,1,1.5)\right| \leq\left(p^{*}, p^{*}, p^{*}\right), \\
\left|\frac{\widetilde{w}_{3}}{\widetilde{w}_{4}}-(1.5,2,2.5)\right| \leq\left(p^{*}, p^{*}, p^{*}\right),\left|\frac{\widetilde{w}_{4}}{\widetilde{w}_{5}}-(1.5,2,2.5)\right| \leq\left(p^{*}, p^{*}, p^{*}\right), \\
\left|\frac{\widetilde{w}_{2}}{\widetilde{w}_{3}}-(0.67,1,1.5)\right| \leq\left(p^{*}, p^{*}, p^{*}\right),\left|\frac{\widetilde{w}_{1}}{\widetilde{w}_{4}}-(1.5,2,2.5)\right| \leq\left(p^{*}, p^{*}, p^{*}\right), \\
\left|\begin{array}{l}
\widetilde{w}_{3} \\
\widetilde{w}_{5}
\end{array}-(3.5,4,4.5)\right| \leq\left(p^{*}, p^{*}, p^{*}\right), \\
\sum_{j=1}^{5} G\left(\widetilde{w}_{j}\right)=1, l_{j} \leq m_{j} \leq u_{j}, l_{j} \geq 0, j=1,2, \ldots, 5 .
\end{gathered}
$$


Then, we can obtain the following optimization model represented by crisp numbers:

$$
\min p^{*}
$$

$$
\begin{gathered}
\left|\frac{l_{2}}{u_{1}}-0.67\right| \leq p,\left|\frac{m_{2}}{m_{1}}-1\right| \leq p,\left|\frac{u_{2}}{l_{1}}-1.5\right| \leq p,\left|\frac{l_{1}}{u_{3}}-0.67\right| \leq p,\left|\frac{m_{1}}{m_{3}}-1\right| \leq p, \\
\left|\frac{u_{1}}{l_{3}}-1.5\right| \leq p,\left|\frac{l_{3}}{u_{4}}-1.5\right| \leq p,\left|\frac{m_{3}}{m_{4}}-2\right| \leq p,\left|\frac{u_{3}}{l_{4}}-2.5\right| \leq p,\left|\frac{l_{4}}{u_{5}}-1.5\right| \leq p, \\
\left|\frac{m_{4}}{m_{5}}-2\right| \leq p,\left|\frac{u_{4}}{l_{5}}-2.5\right| \leq p,\left|\frac{l_{2}}{u_{3}}-0.67\right| \leq p,\left|\frac{m_{2}}{m_{3}}-1\right| \leq p,\left|\frac{u_{2}}{l_{3}}-1.5\right| \leq p, \\
\left|\frac{l_{1}}{u_{4}}-1.5\right| \leq p,\left|\frac{m_{1}}{m_{4}}-2\right| \leq p,\left|\frac{u_{1}}{l_{4}}-2.5\right| \leq p,\left|\frac{l_{3}}{u_{5}}-3.5\right| \leq p,\left|\frac{m_{3}}{m_{5}}-4\right| \leq p, \\
\left|\frac{u_{3}}{l_{5}}-4.5\right| \leq p, \\
(1 / 6)\left(l_{1}+4 m_{1}+u_{1}+l_{2}+4 m_{2}+u_{2}+l_{3}+4 m_{3}+\right. \\
\left.+u_{3}+l_{4}+4 m_{4}+u_{4}+l_{5}+4 m_{5}+u_{5}\right)=1, \\
l_{1} \leq m_{1} \leq u_{1}, l_{2} \leq m_{2} \leq u_{2}, l_{3} \leq m_{3} \leq u_{3}, l_{4} \leq m_{4} \leq u_{4}, l_{5} \leq m_{5} \leq u_{5}, \\
l_{1} \geq 0, l_{2} \geq 0, l_{3} \geq 0, l_{4} \geq 0, l_{5} \geq 0, p \geq 0 .
\end{gathered}
$$

By solving Equation (13), the optimal fuzzy weights of five criteria (quality, price, comfort, safety and style) are calculated as follows:

$$
\begin{aligned}
& \widetilde{w}_{1}^{*}=(0.2169,0.2637,0.3248) ; \widetilde{w}_{2}^{*}=(0.2176,0.2637,0.3254), \\
& \widetilde{w}_{3}^{*}=(0.2167,0.2637,0.3248) ; \widetilde{w}_{4}^{*}=(0.1299,0.1318,0.1445), \\
& \widetilde{w}_{5}^{*}=(0.0578,0.0659,0.0866) ; \widetilde{\chi}^{*}=(0.7532,0.7532,0.7532) .
\end{aligned}
$$

Then, we can obtain the crisp weights (GMIR) of five criteria, which are:

$w_{1}^{*}=0.2661 ; w_{2}^{*}=0.2662 ; w_{3}^{*}=0.2660 ; w_{4}^{*}=0.1336 ; w_{5}^{*}=0.0680$.

It can be seen that price $>$ quality $>$ comfort $>$ safety $>$ style, which is in accordance with the preference order obtained by employing BWM and fuzzy BWM [6]. The consistency ratio is 0.0818 , which is lower than that obtained by using BWM (0.2237) and fuzzy BWM (0.0984). It can be concluded that the fuzzy FUCOM shows higher comparison consistency than the BWM method.

Example 2. The buying company can employ four criteria $C_{i}, i=1,2, \ldots, 4$, where $C_{1}$ is willingness to improve performance, $C_{2}$ - willingness to share information, $C_{3}$ - willingness to rely on each other, and $C_{4}$ - willingness to become involved in a long-term relationship, to evaluate supplier performance. The weights of these four criteria were calculated using BWM and fuzzy BWM [6].

The fuzzy comparative priorities of the criteria are calculated. Willingness to improve performance $\left(C_{1}\right)$ is selected as the best criterion, and willingness to share information $\left(C_{2}\right)$ is regarded as the worst criterion (Table 2).

Table 2. Fuzzy comparative priorities (Example 2)

\begin{tabular}{|c|c|c|c|c|}
\hline Criteria & $C_{1}$ & $C_{3}$ & $C_{4}$ & $C_{2}$ \\
\hline$\tilde{\varphi}_{(k-1) / k}$ & $(1,1,1)$ & $(0.67,1,1.5)$ & $(1,1,1)$ & $(2.5,3,3.5)$ \\
\hline$\tilde{\varphi}_{(k-2) / k}$ & & & $(0.67,1,1.5)$ & $(2.5,3,3.5)$ \\
\hline
\end{tabular}

Stepping on fuzzy group FUCOM for getting the optimal fuzzy weights of all criteria, the following nonlinear constrained optimization problem is built: 
(14)

$$
\begin{gathered}
\min \tilde{\chi}^{*}, \\
\left|\frac{\widetilde{w}_{1}}{\widetilde{w}_{3}}-(0.67,1,1.5)\right| \leq\left(p^{*}, p^{*}, p^{*}\right),\left|\frac{\widetilde{w}_{3}}{\widetilde{w}_{4}}-(1,1,1)\right| \leq\left(p^{*}, p^{*}, p^{*}\right), \\
\left|\frac{\widetilde{w}_{4}}{\widetilde{w}_{2}}-(2.5,3,3.5)\right| \leq\left(p^{*}, p^{*}, p^{*}\right), \sum_{j=1}^{4} G\left(\widetilde{w}_{j}\right)=1, \\
l_{j} \leq m_{j} \leq u_{j}, l_{j} \geq 0, j=1,2, \ldots, 4 .
\end{gathered}
$$
numbers:

Then, we can obtain the following optimization model represented by crisp

$$
\begin{gathered}
\min p^{*}, \\
\left|\frac{l_{1}}{u_{3}}-0.67\right| \leq p,\left|\frac{m_{1}}{m_{3}}-1\right| \leq p,\left|\frac{u_{1}}{l_{3}}-1.5\right| \leq p,\left|\frac{l_{3}}{u_{4}}-1\right| \leq p, \\
\left|\frac{m_{3}}{m_{4}}-1\right| \leq p,\left|\frac{u_{3}}{l_{4}}-1\right| \leq p,\left|\frac{l_{4}}{u_{2}}-2.5\right| \leq p,\left|\frac{m_{4}}{m_{2}}-3\right| \leq p,\left|\frac{u_{4}}{l_{2}}-3.5\right| \leq p, \\
\frac{1}{6}\left(l_{1}+4 m_{1}+u_{1}+l_{2}+4 m_{2}+u_{2}+l_{3}+4 m_{3}+u_{3}+l_{4}+4 m_{4}+u_{4}\right)=1, \\
l_{1} \leq m_{1} \leq u_{1}, l_{2} \leq m_{2} \leq u_{2}, l_{3} \leq m_{3} \leq u_{3} ; l_{4} \leq m_{4} \leq u_{4}, \\
l_{1} \geq 0, l_{2} \geq 0, l_{3} \geq 0, l_{4} \geq 0, p \geq 0 .
\end{gathered}
$$

By solving Equation (15), the optimal fuzzy weights of four criteria are calculated as follows:

$$
\begin{aligned}
& \widetilde{w}_{1}^{*}=(0.1982,0.2973,0.4450), \widetilde{w}_{2}^{*}=(0.0849,0.0990,0.1188), \\
& \widetilde{w}_{3}^{*}=(0.2973,0.2973,0.2973), \widetilde{w}_{4}^{*}=(0.2973,0.2973,0.2973) \text {, } \\
& \tilde{\chi}^{*}=(0.0034,0.0034,0.0034) \text {. }
\end{aligned}
$$

Then, we can obtain the crisp weights (by using GMIR formula) of four criteria, which are:

$$
w_{1}^{*}=0.3054, w_{2}^{*}=0.1000, w_{3}^{*}=0.2973, w_{4}^{*}=0.2973 \text {. }
$$

It can be seen that willingness to improve performance $\left(C_{1}\right)$ is the most important criterion (0.3054) in terms of buyer's evaluation of supplier performance, the next most important criteria are willingness to rely on each other $\left(C_{3}\right)$ and willingness to become involved in a long-term relationship $\left(C_{4}\right)(0.2973)$, and willingness to share information $\left(C_{2}\right)(0.1000)$. This weight order is consistent with results obtained using BWM and fuzzy BWM methods.

The consistency ratio for this case is 0.0012 , which is significantly lower than that of the BWM technique (0.382) and fuzzy BWM method (0.0353). It can be concluded that the fuzzy group FUCOM shows higher priority comparison consistency than the BWM and fuzzy BWM.

\section{Conclusions and future directions}

The presented fuzzy group FUCOM allows team of experts to evaluate in linguistic terms both beneficial and cost criteria. The new method has high efficiency that is achieved by incorporating the following features: 1) a relatively small number of fuzzy pairwise assessments due to the predefined order of criteria; 2) a built-in mechanism for calculating the optimal values of fuzzy relative weights; 3) an effective rule for fuzzy-to-crisp numbers conversion. The defuzzified values of the fuzzy weight coefficients are calculated by Graded Mean Integration Representation formula. This type of relationship (a linear combination) reduces the time complexity without affecting the quality of the solution. Therefore, this new method has the 
potential to solve efficiently weight estimation problems under uncertainty and with vague assessments.

Two practical examples are discussed in order to illustrate the efficiency of the proposed fuzzy group FUCOM with symmetric triangular fuzzy numbers. The results obtained show that the new fuzzy extension is reliable and can successfully solve the task of relative weight determination. In the future, we plan to integrate the proposed fuzzy FUCOM with other comprehensive MCDM methods, such as EDAS, CODAS or MARCOS. We can also attempt to develop other FUCOM extensions via interval value type-2, hesitant, intuitionistic or neutrosophic fuzzy numbers. We will also apply the fuzzy group FUCOM in other real world problems, such as selecting the optimal agriculture and evaluating the benefits of eco-agricultures in a big data environment.

Acknowledgements: This research is supported by European RDF and NSF under grants No BG05M2OP001-1.002-0002 "Digitization of Economy in Big Data Environment" and No KP-06-PN36/2 BG PLANTNET "Establishment of national information network GENEBANK plant genetic resources".

\section{References}

1. B a di, I., A. A b d u $1 \mathrm{~s} \mathrm{~h}$ a h e d. Ranking the Libyan Airlines by Using Full Consistency Method (FUCOM) and Analytical Hierarchy Process (AHP). - Operational Research in Engineering Sciences: Theory and Applications, Vol. 2, 2019, No 1, pp. 1-14.

2. C a o, Q., M. O. E s a n g b e d o, S. B a i, C. O. E s a n g b e d o. Grey SWARA-FUCOM Weighting Method for Contractor Selection MCDM Problem: A Case Study of Floating Solar Panel Energy System Installation. - Energies, Vol. 12, 2019, No 13.

3. Erol, I., S. Sencer, R. Sari. A New Fuzzy Multi-Criteria Framework for Measuring Sustainability Performance of a Supply Chain. - Ecological Economics, Vol. 70, 2011, No 6, pp. 1088-1100.

4. F a n, J. P., Y. J. L i, M. Q. W u. Technology Selection Based on EDAS Cross-Efficiency Evaluation Method. - IEEE Access, Vol. 7, pp. 58974-58980.

5. Fa zlollaht a b a r, H., A. S ma i 1 b a ši ć, Ž. S te vić. FUCOM Method in Group DecisionMaking: Selection of Forklift in a Warehouse. - Decision Making: Applications in Management and Engineering, Vol. 2, 2019, No 1, pp. 49-65.

6. Guo, S., H. Zha o. Fuzzy Best-Worst Multi-Criteria Decision-Making Method and Its Applications. - Knowledge-Based Systems, Vol. 121, 2017, pp. 23-31.

7. I li eva, G. Decision Making Methods in Agent Based Modelling. - In: CEUR Workshop Proceedings 752, 2011, pp. 8-17.

8. I 1 i e v a, G. Group Decision Analysis Algorithms with EDAS for Interval Fuzzy Sets. - Cybernetics and Information Technologies, Vol. 18, 2018, No 2, pp. 51-64.

9. Mardani, A., E. K. Zavadskas, Z. Khali fah, N. Zakuan, A. Jusoh, K. M. Nor, M. Khoshnoudi. A Review of Multi-Criteria Decision-Making Applications to Solve Energy Management Problems: Two Decades from 1995 to 2015. - Renewable and Sustainable Energy Reviews, Vol. 71, 2017, pp. 216-256.

10. N a g o o r, G a n i, A., S. N. M o h a m e d A s s a r u d e e n. A New Operation on Triangular Fuzzy Number for Solving Fuzzy Linear Programming Problem. - Applied Mathematical Sciences, Vol. 6, 2012, No 12, pp. 525-532.

11. N a z, S., M. A k r a m. Novel Decision-Making Approach Based on Hesitant Fuzzy Sets and Graph Theory. - Computational and Applied Mathematics, Vol. 38, 2019, No 7, pp. 1-26.

12. P a m u č a r, D., M. D e v e c i, F. C a n 1 t e z, D. B o z a n i c. A Fuzzy Full Consistency MethodDombi-Bonferroni Model for Prioritizing Transportation Demand Management Measures. Applied Soft Computing, Vol. 87, 2020. 
13. P a m u č a r, D., Ž. S t e vić, S. S r e m a c. New Model for Determining Weight Coefficients of Criteria in MCDM Models: Full Consistency Method (FUCOM). - Symmetry, Vol. 10, 2018, No 9.

14. P e n e va, V., I. P o p che v. Fuzzy Criteria Importance Depending on Membership Degrees of Fuzzy Relations. - Compt. Rend. Acad. bulg. Sci., Vol. 61, 2008, No 5, pp. 579-584.

15. Peneva, V., I. Popchev. Multicriteria Decision Making Based on Fuzzy Relations. Cybernetics and Information Technologies, Vol. 8, 2008, No 4, pp. 3-12.

16. P e n e va, V., I. P o p c h e v. Multicriteria Decision Making by Fuzzy Relations and Weighting Functions for the Criteria. - Cybernetics and Information Technologies, Vol. 9, 2009, No 4, pp. 58-71.

17. P o p che v, I., V. P e n e va. A Fuzzy Multicriteria Decision Making Algorithm. - In: Proc of 10th International Conference on Multiple Criteria Decision Making, 19-24 July 1992, Taipei, Vol. II, pp. 11-16.

18. R a d e v a, I. Multicriteria Fuzzy Sets Application in Economic Clustering Problems. - Cybernetics and Information Technologies, Vol. 17, 2017, No 3, pp. 29-46.

19. S h a k e e $1, \mathrm{M}, \mathrm{S}$. A b d u $11 \mathrm{a}$ h. Some Induced Interval-Valued Pythagorean Trapezoidal Fuzzy Averaging Aggregation Operators Based on Einstein Operations and Their Application in Group Decision-Making. - Computational and Applied Mathematics, Vol. 38, 2019.

20. S h araf, I. M. An Interval Type-2 Fuzzy TOPSIS Using the Extended Vertex Method for MAGDM. - SN Applied Sciences, Vol. 2, 2020.

21. S h a r a f, I. M. TOPSIS with Similarity Measure for MADM Applied to Network Selection. Computational and Applied Mathematics, Vol. 37, 2018, No 4, pp. 4104-4121.

22. S te vić, Z., N. B rk ović. A Novel Integrated FUCOM-MARCOS Model for Evaluation of Human Resources in a Transport Company. - Logistics, Vol. 4, 2020, No 1.

23. T a b a t ab a e i, M. H., M. A miri, M. Ghahre man 1 oo, M. Ke sh av ar z-Ghor ab a ee, E. K. Z a vad ska s, J. A n t u che vi cien e. Hierarchical Decision-Making Using a new Mathematical Model Based on the Best-Worst Method. - International Journal of Computers Communications \& Control, Vol. 14, 2019, No 6, pp. 669-684.

24. V a n La arh o ve n, P. J. M., W. P e d r y c z. A Fuzzy Extension of Saaty's Priority Theory. Fuzzy Sets and Systems, Vol. 11, 1983, No 1-3, pp. 229-241.

25. W u, W. W., Y. T. L e e. Developing Global Managers' Competencies Using the Fuzzy DEMATEL Method. - Expert Systems with Applications, Vol. 32, 2007, No 2, pp. 499-507.

26. Z h a o, H., S. G u o. Selecting Green Supplier of Thermal Power Equipment by Using a Hybrid MCDM Method for Sustainability. - Sustainability, Vol. 6, 2014, No 1, pp. 217-235.

Received: 20.03.2020; Second Version: 07.05.2020; Accepted: 21.05.2020 